natural hormones have been produced in the laboratory and made available for the treatment of disease. Thus diethylstilbœstrol is a synthetic oestrogen which is active by mouth and is already finding a place in treatment. A lengthy account of the physiological action of different hormones is given in the section of the report devoted to work carried out at the National Institute.

This summary is intended only to give a general idea of the work and policy of the Medical Research Council : brief reference has also been made to a few subjects in which there have been recent advances in knowledge. The interested reader will find in the report full references to all the researches carried out under the auspices of the Council, which have been published in the scientific journals, as well as mention of the many investigations still in progress.

\title{
Observation of the Sun's Corona and Prominences without Eclipses
}

M BERNARD LYOT, of the Astrophysical 1. Section of the Paris Observatory, delivered the George Darwin Lecture before the Royal Astronomical Society on May 12, the subject being "A Study of the Solar Corona and Prominences without Eclipses". To this research, M. Lyot has made unique and fundamental contributions for which he has been awarded this year the Royal Astronomical Society's Gold Medal. The presentation of the Medal was made prior to the lecture by the president of the Society, Prof. H. C. Plummer, who spoke of the medallist's great achievement in recording for the first time the sun's corona without an eclipse.

M. Lyot began his lecture with a brief review of past attempts made by various investigators from 1878 onwards to record the solar corona without a total eclipse of the sun. Direct photography from high altitude sites, thermo-electric, spectroscopic and polariscopic methods had all been tried but gave negative results. In consequence, the problem was generally regarded as being beyond the range of observational technique. Ten years ago, M. Lyot, encouraged by the advice of the late Prof. H. O. Barnard of the Colombo Observatory, made a fresh attempt to record the corona in full sunlight.

Measurements made during total solar eclipses showed that the brightness of the corona at $2^{\prime}$ from the sun's edge was only about one-millionth that of the light from the disk. To obtain any success, it was therefore a primary necessity to reduce to a negligible amount the diffusion of light by the optical train of the telescope. This diffused light in an ordinary telescope is at least two hundred times greater than the inner coronal image. Furthermore, the whitish aureole of diffracted light around the sun produced by dust in the earth's lowest atmosphere and by ice crystals of cirrus cloud is generally in itself more than a hundred times brighter than the corona.
To overcome the first difficulty, M. Lyot constructed with increasing success three special coronographs. To avoid the dust, he took his apparatus to the Pic du Midi in the Pyrenees at a height of 2,870 metres, where excellent conditions on cloudless days are obtained especially in the late spring.

The general plan on which the coronographs were constructed is as follows: A single planoconvex lens $(A)^{*}$ forms an image of the sun on a blackened disk of brass projecting just beyond the image by $15^{\prime \prime}-20^{\prime \prime}$. A field lens placed behind the disk produces an image of the lens $A$ in a diaphragm, the edge of which intercepts the light diffracted by $A$. A small screen placed in the centre of the diaphragm intercepts the light of the solar image produced by reflection from the surfaces of $A$. Behind the diaphragm and the screen, protected from the diffused light, an overcorrected objective forms an achromatic image of the corona on the plate. The whole optical train is contained in a tube, open only during the observations and coated inside with thick oil to trap the particles of dust. The primary lens must be wiped frequently with particular care.

M. Lyot showed a number of slides of the observatory and instruments. Even under the best conditions, the last part of the ascent of the Pic du Midi has to be made on foot. The coronographs are made to telescope into sections for manual transport often over deep snow.

\section{The Corona: Direct Photographs}

Lyot's work on the direct photography of the corona was made in two stages : (1) 1930-34, when the new method by his coronographs was being

* The two later objectives were cut from flawless disks of borosilicate crown glass produced by Messrs. Parra-Mantois. The lens of the second coronograph was ground in the Institut d'optique in Paris, while that of the third instrument was figured by $M$. Couder at the Paris Observatory. 
tested; (2) after 1934, when new results were attempted. The first positive result was obtained on July 25, 1930, with his coronograph of $8 \mathrm{~cm}$. aperture (stopped down to $3 \mathrm{~cm}$.) and 2 metres in focal length. Using a red colour-filter, prominences were easily seen. The sun's image was surrounded by a slight halo without detail. Polarimeter tests showed the light of this halo to be polarized in a radial plane like the corona as observed at total eclipses but less strongly. The measures, in point of fact, indicated that one fifth of the light of this halo belonged to the corona, the remainder being due to diffusion. But there was other evidence to prove that Lyot had in reality isolated the sun's inner corona. On July 30 and the following days, an attached spectroscope showed amongst the absorption lines due to diffused solar light, an emission line in the green the wave-length of which to within one angstrom was the same as that of the well-known coronal line, $\lambda$ 5303. The red coronal line, $\lambda 6374$, was also faintly visible.

A year later, a larger and more perfect coronograph $(13 \mathrm{~cm}$. in aperture and a focal length of 3.15 metres) had been constructed and transported with accessory apparatus to the Pic du Midi. With this instrument the first direct photograph of the sun's corona was obtained on July 21, 1931. This photograph and others exhibited on the screen showed the distinctive features of the inner corona as photographed at total eclipses-luminous arches, dark spaces, fine jets of coronal matter, etc. Changes of structure were also apparent within 24 hours. To this coronograph was attached a spectroheliograph, isolating a part of the spectrum $10 \mathrm{~A}$. wide containing the green line 5303. Thus the distribution and strength of this coronal line all around the sun could be studied.

Slides of the earliest direct photographs of the corona had to be made by successive copying on the same plate, and in exact superposition, of the six best of twelve negatives taken in succession on any one day, the coronograph being turned on its axis through $30^{\circ}$ between each exposure. This procedure diminished considerably the effect of spots produced by dust particles deposited on the second lens of the coronograph. In 1938, a rotatory mechanism was added to the latest of Lyot's coronographs $(20 \mathrm{~cm}$. in aperture and 4 metres in focal length) which annuls the effect of dust particles on the lens. A single negative is sufficient to gain a good picture of the corona (on a scale of $65 \mathrm{~mm}$. to the solar diameter) with an exposure as short as seven seconds.

Among the many slides shown by M. Lyot to illustrate the delicate, complex structure of the inner corona and its changes, were some taken on May 11, 1937, when Mercury passed close to the sun's disk. At 10h. 10m., when the planet was only $1^{\prime} 11^{\prime \prime}$ from the sun's edge, it was seen projected against a prominence. Later, at $11 \mathrm{~h} .14 \mathrm{~m}$., when at a distance of $2^{\prime} 37^{\prime \prime}$, it was outlined against the corona itself; the sky then clouded.

\section{The Coronal Spectrum}

New and powerful spectrographs were devised by Lyot to be used with his two latest coronographs, and with these the spectrum of the corona has been explored from $3350 \mathrm{~A}$. (the limit of the transparency of the coronographs) to $1200 \mathrm{~A}$., which represents the limit of the sensitivity of the plates. To compass this range of spectrum, seven different kinds of plates had to be used, with exposures ranging from 10 minutes in the visible spectrum to $\mathbf{4}$ hours in the infra-red. M. Lyot then proceeded to show examples of the two hundred or so spectra which he has obtained up to the present time. The slit of the spectrograph is in the form of an arc crossing the east and west sides of the corona about $40^{\prime \prime}$ from the sun's edge between latitudes $\pm 70^{\circ}$. These spectra, usually on a scale of 5-7 $\frac{1}{2} \mathrm{~A}$. per mm., show in addition to a continuous spectrum from the corona (and also the spectra of any prominences present), eleven coronal emission lines of which five are new lines not previously recorded under conditions of total solar eclipse. These emission lines are as follows, the new lines being indicated by an asterisk:

$\begin{array}{lc}3388 \mathrm{~A} . & 7060 \mathrm{~A} .^{*} \dagger \\ 5116 & 7892 \\ 5303 & 8024^{*} \\ 5694^{*} & 10747^{*} \\ 6374 & 10798^{*} \\ 6702 & \end{array}$

$\dagger$ Found later by Menzel on spectrograms taken at the eclipse of 1936 .

The wave-length of the fourth and seventh lines listed are given more exactly as $5694.42 \mathrm{~A}$. and $7059 \cdot 62 \mathrm{~A}$.

A notable feature of all the coronal lines shown is their great width. Allowing for instrumental corrections, their average widths are $0.8 \mathrm{~A}$. in the green, $0 \cdot 9-1 \cdot 0 \mathrm{~A}$. in the red, and greater than $1 \mathrm{~A}$. in the infra-red. The distribution of these lines around the sun show differences from one another which are being studied. As an example, M. Lyot cited the curious distribution of $\lambda 5694$, which is invisible where $\lambda 5303$ is at a maximum. Another anomaly is the absence from the above list of the line $\lambda 5536$, of which no trace appeared on spectrograms taken in 1936, 1937 and 1938 under very favourable conditions and high solar activity. Perhaps the line does not extend to $40^{\prime \prime}$ above the sun's limb like the other lines. Lyot considers 
that the eleven lines listed fall into three groups: (a) $\lambda \lambda 5303,3388,5116,6702,7059,8024$, and probably 10747 and $10798 ;(b) 5694 ;(c) 6374$ and 7892.

By measuring the differential Doppler effect given by the strong emission line, $5303 \mathrm{~A}$., at the east and west edges of the sun, it is found that the corona rotates in the same sense as the general body of the sun and at approximately the same rate.

\section{SPeCtra of Prominences}

Some of the Pic du Midi spectrograms are very rich in lines from the spectra of prominences. The hydrogen spectrum was shown represented by the Paschen series (from the 6th term at 10939 A. to the 3lst term, and the Balmer series in the ultraviolet from the 8 th also to the 31st term. The lines of the Paschen series are much fainter than those of the Balmer series, but the atmospheric diffusion, greatly reduced in the infra-red, allows spectra of the Paschen series to be obtained easily without an eclipse.

\section{Prominences Through Colour Filters}

The continuous spectrum of prominences has been observed during eclipses, but it was thought that the spectrum was emitted only by some particular parts of the most intense prominences. To test this, three filters were used transmitting (a) continuous spectrum from $6100 \mathrm{~A}$. to $6400 \mathrm{~A}$.; (b) the $D_{3}$ line of helium ; (c) $H \lambda$ of hydrogen. It was seen that $(a)$ and $(b)$ gave identical pictures both with respect to form and relative intensities. These results, taken in conjunction with polarization measurements, indicate that this continuous spectrum comes from all parts of a prominence, and in the visible range would appear to be due to the diffusion of the sun's light by the electrons that they contain.

\section{Movements of Prominences}

Lyot's coronograph, supplied with a filter isolating the line $H \lambda$, shows the prominences with great brilliancy against a very dark sky, and their faintest details appear. Since 1935, the movements of stable and eruptive prominences have been recorded at the Pic du Midi on cinematograph films, usually at a rate of two per minute and, in projection, speeded up 600 times. The height of the pictures which M. Lyot showed usually represented about $380,000 \mathrm{~km}$., that is the distance of the moon from the earth, or thirty times the earth's diameter. These gave an extraordinary exhibition of prominence movements, of which a few examples may be outlined :

(1) Filaments fading away as they rise and brightening again as they fall back into the sun with a speed of $180 \mathrm{~km}$. $/ \mathrm{sec}$.

(2) A suspended cloud pours luminous material inwards to the chromosphere.

(3) A fountain-like prominence is suddenly thrown up by an explosion.

(4) A particularly stable prominence; small internal motions are, however, perceptible. After many hours, part of the cloud becomes very bright. A chromospheric eruption leaves the sun at the speed of $700 \mathrm{~km}$. per sec. and cuts the prominence into two parts. The luminous mass, its equilibrium lost, flows into the sun and seems to flow around an invisible obstacle.

(5) A prominence similar to those called "coronal prominences" by $\mathrm{Pe}^{\wedge}$ tit and McMath. Material, originating in invisible clouds, flows downwards on both sides of a loop or arch into the chromosphere.

In conclusion, M. Lyot said that it would be very interesting to compare the observations of the corona by both methods-with and without eclipses. The outer parts of the corona can be obtained only during eclipses; the new method without an eclipse shows only the inner corona, but daily or even hourly variations of the corona can be recorded. With regard to the coronal spectrum; in the ultra-violet, the violet and the blue, the atmospheric diffusion is very strong, and the new method is in consequence very inferior to the old method. In the green, the diffusion is less strong, and its effects can be diminished by increasing the dispersion and making longer exposures. By choosing days on which the corona is intense, the faint coronal lines appear. The numerous spectrograms on a large scale, possible with the new method, enable the lines to be distinguished from the agglomerations of grains on the plate, and their wave-lengths are measurable with great precision. In the infra-red the new method is superior.

For studying prominences, the extra-eclipse method alone enables the solar observer to wait for the appearance of very bright objects having the richest and most interesting spectra. Observations made without eclipses can therefore not replace the work of expeditions investigating total eclipse phenomena, but the two methods complement one another in numerous ways, and their simultaneous use would increase our knowledge more rapidly of the sun's outer atmosphere. 